\title{
Relating leptogenesis parameters to light neutrino masses
}

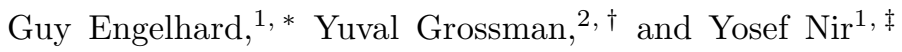 \\ ${ }^{1}$ Department of Particle Physics, Weizmann Institute of Science, Rehovot 76100, Israel \\ ${ }^{2}$ Department of Physics, Technion-Israel Institute of Technology, Technion City, Haifa 32000, Israel
}

(Dated: October 11, 2018)

\begin{abstract}
We obtain model independent relations among neutrino masses and leptogenesis parameters. We find exact relations that involve the CP asymmetries $\epsilon_{N_{\alpha}}$, the washout parameters $\tilde{m}_{\alpha}$ and $\theta_{\alpha \beta}$, and the neutrino masses $m_{i}$ and $M_{\alpha}$, as well as powerful inequalities that involve just $\tilde{m}_{\alpha}$ and $m_{i}$. We prove that the Yukawa interactions of at least two of the heavy singlet neutrinos are in the strong washout region $\left(\tilde{m}_{\alpha} \gg 10^{-3} e V\right)$.
\end{abstract}

Introduction. Singlet neutrinos with heavy Majorana masses and with Yukawa couplings to the active neutrinos generate light neutrino masses via the see-saw mechanism and a baryon asymmetry via leptogenesis [1], providing attractive qualitative solutions to these two important puzzles. To be quantitatively successful, the seesaw mechanism should lead to the two observed mass scales,

$$
\begin{aligned}
& m_{s} \equiv\left(\Delta m_{\mathrm{sol}}^{2}\right)^{1 / 2} \sim 0.009 \mathrm{eV}, \\
& m_{a} \equiv\left(\Delta m_{\mathrm{atm}}^{2}\right)^{1 / 2} \sim 0.05 \mathrm{eV},
\end{aligned}
$$

while leptogenesis should lead to the value extracted from observations,

$$
Y_{\mathcal{B}}^{\mathrm{obs}} \equiv \frac{n_{B}-n \bar{B}}{s}=(8.7 \pm 0.3) \times 10^{-11} .
$$

Unfortunately, because the leptogenesis parameters - the CP asymmetries and the washout factors - directly involve the heavy singlet neutrinos, we cannot realistically hope that they will be measured. In order to make further progress in the investigation of leptogenesis, it is highly desirable to relate the leptogenesis parameters to measurable mass parameters. The purpose of this work is to obtain such relations.

The relations that we obtain involve the washout parameters of all the heavy singlet neutrinos $N_{\alpha}$. While most leptogenesis studies have focussed on the contributions from the decays of $N_{1}$, the lightest heavy singlet, it has been realized that, in general, the contributions from the decays of the heavier singlet neutrinos must not be neglected [2, 3, 4]. Indeed, our results reinforce this statement.

Notations. The relevant Lagrangian terms involve, in addition to the $N_{\alpha}$ 's, the light lepton $\mathrm{SU}(2)$-doublets $L_{i}$ and $\mathrm{SU}(2)$-singlets $E_{i}(i=e, \mu, \tau$ is a flavor index), and the standard model Higgs $H$ :

$$
-\mathcal{L}=\frac{1}{2} M_{\alpha} N_{\alpha} N_{\alpha}+\lambda_{\alpha i} H N_{\alpha} L_{i}+Y_{i} H^{\dagger} L_{i} E_{i} .
$$

Eq. (3) is written in the mass basis for the singlet neutrinos and for the charged leptons, that is, $M$ and $Y$ are diagonal.
The light neutrino mass matrix is given by

$$
m_{\nu}=v^{2} \lambda^{T} M^{-1} \lambda
$$

where $v=\langle H\rangle$. Reversing this relation, one can express the Yukawa couplings $\lambda_{\alpha i}$ in terms of the diagonal mass matrix $M$, the matrix $m=\operatorname{diag}\left(m_{1}, m_{2}, m_{3}\right)$ (where $m_{i}^{2}$ are the eigenvalues of $m_{\nu} m_{\nu}^{\dagger}$ ), the leptonic mixing matrix $U$ and an orthogonal complex matrix $R$ [5]:

$$
\lambda=\frac{1}{v} M^{1 / 2} R m^{1 / 2} U^{\dagger} .
$$

The baryon number generated from the decays of the $N_{\alpha}$ neutrinos can be written as follows:

$$
Y_{\mathcal{B}}=-1.4 \times 10^{-3} \sum_{\alpha, \beta} \epsilon_{N_{\alpha}} \eta_{\alpha \beta},
$$

where $\epsilon_{N_{\alpha}}$ is the CP asymmetry generated in $N_{\alpha}$ decays:

$$
\epsilon_{N_{\alpha}}=\frac{\Gamma\left(N_{\alpha} \rightarrow \ell H\right)-\Gamma\left(N_{\alpha} \rightarrow \bar{\ell} \bar{H}\right)}{\Gamma\left(N_{\alpha} \rightarrow \ell H\right)+\Gamma\left(N_{\alpha} \rightarrow \bar{\ell} \bar{H}\right)},
$$

and $\eta_{\alpha \beta}$ denotes the efficiency factor related to the washout of the asymmetry $\epsilon_{N_{\alpha}}$ due to $N_{\beta}$ interactions. (If leptogenesis takes place at $T \lesssim 10^{12}$, flavor indices should be added [2, 6, 7, 8].) It is convenient for our purposes to further define a matrix of dimensionful quantities $\tilde{m}_{\alpha \beta}$ :

$$
\tilde{m}=v^{2} M^{-1 / 2} \lambda \lambda^{\dagger} M^{-1 / 2}
$$

Note that $\tilde{m}$ is a positive matrix and, in particular, $\left|\tilde{m}_{\alpha \beta}\right|^{2} \leq \tilde{m}_{\alpha \alpha} \tilde{m}_{\beta \beta}$. In terms of the parametrization (5), we have

$$
\tilde{m}_{\alpha \beta}=\sum_{i} m_{i} R_{\alpha i} R_{\beta i}^{*}
$$

In a large part of the parameter space, the washout factors $\eta_{\alpha \alpha}$ depend on the mass and the couplings of $N_{\alpha}$ only via the combination $\tilde{m}_{\alpha} \equiv \tilde{m}_{\alpha \alpha}$ [9]. For example, 
for $M_{1} \ll 10^{14} \mathrm{GeV}$ and $\tilde{m}_{\alpha} \gg m_{*}=2.2 \times 10^{-3} \mathrm{eV}$, we have [10]

$$
\eta_{\alpha \alpha} \approx\left(\frac{5.5 \times 10^{-4} \mathrm{eV}}{\tilde{m}_{\alpha}}\right)^{1.16}
$$

When we talk in this work about the "washout parameters" we refer mainly to the $\tilde{m}_{\alpha}$ 's. The off-diagonal terms in $\tilde{m}$ do, however, play important roles in leptogenesis. First, the CP asymmetries depend on $\mathcal{I} m\left(\tilde{m}_{\alpha \beta}\right)$ (see, for example, Eq. (22) below). Second, $\left|\tilde{m}_{\alpha \beta}\right|$ determines the overlap between the lepton doublet states $\ell_{\alpha}$ and $\ell_{\beta}$ to which $N_{\alpha}$ and $N_{\beta}$ decay, respectively [4]:

$$
\begin{aligned}
\left|\ell_{\alpha}\right\rangle & =\left(\lambda \lambda^{\dagger}\right)_{\alpha \alpha}^{-1 / 2} \sum_{i} \lambda_{\alpha i}\left|\ell_{i}\right\rangle, \\
\cos ^{2} \theta_{\alpha \beta} & \equiv\left|\left\langle\ell_{\alpha} \mid \ell_{\beta}\right\rangle\right|^{2}=\left|\tilde{m}_{\alpha \beta}\right|^{2} /\left(\tilde{m}_{\alpha} \tilde{m}_{\beta}\right) .
\end{aligned}
$$

For the case of strong hierarchy between the masses and the lifetimes of, say, $N_{1}$ and $N_{2}$, and $\tilde{m}_{1} \gg m_{*}$, the interactions of $N_{1}$ first project $\epsilon_{N_{2}}$ on the directions aligned with or orthogonal to $\ell_{1}$ and then washout the asymmetry in the $\ell_{1}$ direction [4]. For this case, we use an approximate expression for the total lepton asymmetry generated in $N_{2}$ and $N_{1}$ decays:

$Y_{\mathcal{B}} \approx-1.4 \times 10^{-3}\left[\epsilon_{N_{1}} \eta_{11}+\epsilon_{N_{2}} \eta_{22}\left(\cos ^{2} \theta_{12} \eta_{11}+\sin ^{2} \theta_{12}\right)\right]$.

The basic relations. The key point for our results is that $\tilde{m}^{*} \tilde{m}$ and $m_{\nu} m_{\nu}^{\dagger}$ are similar. In particular, the following three relations hold:

$$
\begin{aligned}
\operatorname{det}\left(m_{\nu} m_{\nu}^{\dagger}\right) & =\operatorname{det}\left(\tilde{m}^{*} \tilde{m}\right) \\
\operatorname{Sym}_{2}\left(m_{\nu} m_{\nu}^{\dagger}\right) & =\operatorname{Sym}_{2}\left(\tilde{m}^{*} \tilde{m}\right) \\
\operatorname{Tr}\left(m_{\nu} m_{\nu}^{\dagger}\right) & =\operatorname{Tr}\left(\tilde{m}^{*} \tilde{m}\right)
\end{aligned}
$$

where $\operatorname{Sym}_{2}(A)=\frac{1}{2}\left\{[\operatorname{Tr}(A)]^{2}-\operatorname{Tr}\left(A^{2}\right)\right\}$.

These equations can be written as exact relations involving the light neutrino masses $m_{i}$, the washout parameters $\tilde{m}_{\alpha}$, and the off-diagonal terms $\tilde{m}_{\alpha \beta}$. The latter can be expressed in terms of the CP asymmetries $\epsilon_{N_{\alpha}}$ and the projections $\cos ^{2} \theta_{\alpha \beta}$.

These equalities [as well as the explicit form (9)] can be further used to obtain simple inequalities involving only the washout parameters $\tilde{m}_{\alpha}$ and the light neutrino masses $m_{i}$. In particular, we are able to show that some (and in some cases all) of the $N_{\alpha}$ interactions are in the strong washout region.

We note that there is no additional information for us in the leptonic mixing angles. The reason is that $\tilde{m}$ is independent of the mixing angles. This can be seen by noting that $\lambda \lambda^{\dagger}$ is independent of $U$, Eq. (5), or directly from Eq. (9).

We apply Eqs. (13) to two cases, differing in the number of singlet neutrinos $N_{\alpha}$ that are added to the SM. In the " $3+2$ " framework, two such neutrinos are assumed to be relevant to the see-saw mechanism and to leptogenesis, while in the " $3+3$ " framework, there are three. The $3+2$ case is actually a special limit of the $3+3$ framework. When one of the three $\tilde{m}_{\alpha} \rightarrow 0$ (that is, $\left(\lambda \lambda^{\dagger}\right)_{\alpha \alpha} \rightarrow 0$ and/or $\left.M_{\alpha} \rightarrow \infty\right)$, the corresponding $N_{\alpha}$ becomes irrelevant to both the see-saw mechanism and leptogenesis, and the model reduces to effectively a $3+2$ model. We checked that all our results in the $3+3$ framework indeed reproduce the $3+2$ results in this limit.

We do not consider $3+m$ models with $m>3$ because, in general, they are similar to the $3+3$ model. To understand that, note that $\ell_{2}\left(\ell_{3}\right)$ has, in general, a component that is orthogonal to $\ell_{1}\left(\ell_{1}\right.$ and $\left.\ell_{2}\right)$. Consequently, part of the asymmetries generated by the decays of $N_{2}$ and $N_{3}$ is protected against washout [4]. The light flavor space is, however, three dimensional and therefore spanned, in general, by $\ell_{1}, \ell_{2}$ and $\ell_{3}$. Consequently, there is no component in $\ell_{\alpha>3}$ that is orthogonal to all three, and the asymmetries $\epsilon_{N_{\alpha>3}}$ are expected to be washed out.

The $3+2$ framework. When we have only two singlet neutrinos $[\alpha=1,2$ in Eq. (3) $]$, one of the light mass eigenvalues vanishes and the other two mass eigenvalues are fixed by phenomenology to one of two discrete possibilities, either normal hierarchy $(\mathrm{NH})$ or inverted hierarchy $(\mathrm{IH})$ :

$$
\begin{aligned}
& m_{1}=0, \quad m_{2}=m_{s}, \quad m_{3}=m_{a} \quad(\mathrm{NH}), \\
& m_{1}=0, \quad m_{2,3} \approx m_{a}, \quad m_{3}-m_{2}=m_{s}^{2} /\left(2 m_{a}\right)
\end{aligned}
$$

Using Eqs. (13), we have

$$
\begin{aligned}
m_{2} m_{3} & =\tilde{m}_{1} \tilde{m}_{2}-\left|\tilde{m}_{12}\right|^{2} \\
m_{2}^{2}+m_{3}^{2} & =\tilde{m}_{1}^{2}+\tilde{m}_{2}^{2}+2 \mathcal{R} e\left(\tilde{m}_{12}^{2}\right) .
\end{aligned}
$$

Using Eqs. (15) and (16), we obtain two inequalities:

$$
\begin{aligned}
\tilde{m}_{2}+\tilde{m}_{1} & \geq m_{3}+m_{2} \\
\left|\tilde{m}_{2}-\tilde{m}_{1}\right| & \leq m_{3}-m_{2}
\end{aligned}
$$

We derived various additional inequalities:

$$
\begin{aligned}
\tilde{m}_{1,2} & \geq m_{2}, \\
\tilde{m}_{1} \tilde{m}_{2} & \geq m_{2} m_{3}, \\
m_{2} / m_{3} \leq \tilde{m}_{1} / \tilde{m}_{2} & \leq m_{3} / m_{2} .
\end{aligned}
$$

(The inequality $\tilde{m}_{1} \geq m_{2}$ was derived in ref. [11].) However, Eqs. (19)-(21) are redundant when the constraints (17) and (18) are imposed.

The combination of the inequalities (17) and (18), together with the known values of $m_{2}$ and $m_{3}$ [Eq. (14)], constrains the allowed region in the $\tilde{m}_{1}-\tilde{m}_{2}$ plane in a very significant way. We plot these constraints in Fig. 1. In particular, we can draw the following conclusions:

1. For NH, both $\tilde{m}_{\alpha}$ are above $m_{s}$ and at least one of them is above $m_{a} / 2$. The two washout factors are within a factor of $m_{a} / m_{s} \sim 6$ of each other. 


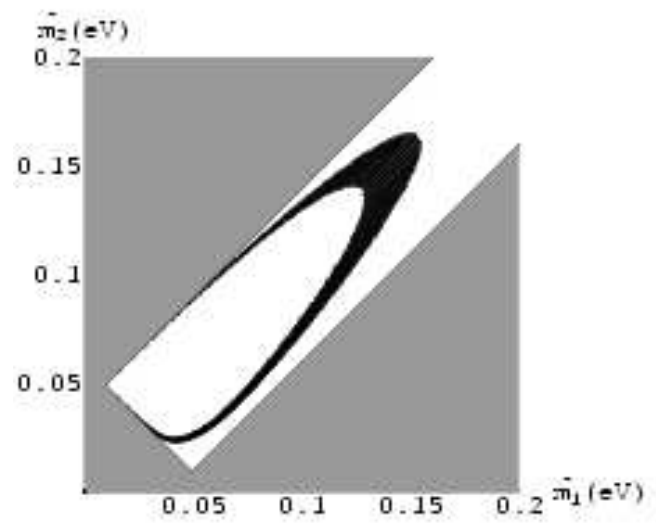

FIG. 1: The constraints in the $\tilde{m}_{1}-\tilde{m}_{2}$ plane in the $3+2$ case with normal hierarchy. The grey region is forbidden by Eqs. (17) and (18). The black region is derived by scanning the parameter space (fixing $M_{1}=10^{12} \mathrm{GeV}$ and $M_{2} / M_{1}=10$ ) and requiring that the resulting baryon asymmetry would be within the $3 \sigma$ range [Eq. (2)].

2. For IH, both $\tilde{m}_{\alpha}$ are above $m_{a}$, while the difference between them is very small, $\leq m_{s}^{2} /\left(2 m_{a}\right)$.

3. In either case, both $N_{2}$ interactions and $N_{1}$ interactions are in the strong washout regime.

As concerns the two CP asymmetries, $\epsilon_{N_{1,2}}$, they can be written as $\left(x_{12} \equiv M_{1} / M_{2}\right)$

$$
\epsilon_{N_{\alpha}}=f_{\alpha}\left(x_{12}\right) \frac{M_{\alpha} \mathcal{I} m\left(\tilde{m}_{12}^{2}\right)}{v^{2} \tilde{m}_{\alpha}} .
$$

The functions $f_{\alpha}\left(x_{12}\right)$ can be found in the literature [12]. Instead of the inequalities (17) and (18), one can combine Eqs. (15), (16) and (22) to obtain an exact relation between the neutrino masses, the washout parameters and the CP asymmetries:

$$
\begin{aligned}
& 4\left(\tilde{m}_{1} \tilde{m}_{2}-m_{2} m_{3}\right)^{2}-\left(\tilde{m}_{1}^{2}+\tilde{m}_{2}^{2}-m_{2}^{2}-m_{3}\right)^{2} \\
& \quad=4 \frac{v^{4} \tilde{m}_{1}^{2} \epsilon_{N_{1}}^{2}}{M_{1}^{2}\left[f_{1}\left(x_{12}\right)\right]^{2}}=4 \frac{v^{4} \tilde{m}_{2}^{2} \epsilon_{N_{2}}^{2}}{M_{2}^{2}\left[f_{2}\left(x_{12}\right)\right]^{2}} .
\end{aligned}
$$

The $3+2$ framework with strong $M_{2} / M_{1}$ hierarchy. While our main focus here is on model independent relations, we can gain some further understanding by assuming mass hierarchy between the two singlet neutrinos. (It also explains features of the black region in Fig. 1 which corresponds to $M_{2} / M_{1}=10$.) In the hierarchical case $\left(x_{12} \ll 1\right)$, we have

$$
\begin{aligned}
f_{1}\left(x_{12}\right) & =-3 /(16 \pi), \\
f_{2}\left(x_{12}\right) & =-x_{12}^{2}\left[\ln \left(x_{12}\right)+1\right] /(4 \pi), \\
\frac{\epsilon_{N_{2}}}{\epsilon_{N_{1}}} & =-\frac{4}{3} \frac{\tilde{m}_{1}}{\tilde{m}_{2}} x_{12}\left(\ln \frac{1}{x_{12}}-1\right) .
\end{aligned}
$$

Using Eqs. (6) and (10), we can give a rough estimate of the ratio between the respective contributions to $Y_{\mathcal{B}}$ :

$$
\frac{\left|\epsilon_{N_{2}}\right| / \tilde{m}_{2}}{\left|\epsilon_{N_{1}}\right| / \tilde{m}_{1}} \sim \frac{\tilde{m}_{1}^{2}}{\tilde{m}_{2}^{2}} \frac{M_{1}}{M_{2}}\left(\ln \frac{M_{2}}{M_{1}}-1\right) .
$$

We would like to emphasize the following points:

1. For a mild hierarchy between $M_{1}$ and $M_{2}, N_{2^{-}}$ leptogenesis must not be neglected. (In this case, $\eta_{12}$ and $\eta_{21}$ have to be taken into account.)

2. In the $\mathrm{NH}$ case, only for a very strong hierarchy, $M_{2} / M_{1} \gg 10^{2}$, it is guaranteed that $N_{1}$ leptogenesis dominates. For IH, the contribution from $\epsilon_{N_{1}}$ is always larger.

3. Given that $\epsilon_{N_{2}}$ and $\epsilon_{N_{1}}$ have opposite signs, partial (and potentially significant) cancellation between the two contributions is quite possible.

In the $3+3$ framework, when the heavy neutrino masses are strongly hierarchical, $\epsilon_{N_{1}}$ is subject to an upper bound 13, 14]:

$$
\left|\epsilon_{N_{1}}\right| \leq \epsilon^{\mathrm{DI}} \equiv \frac{3}{16 \pi} \frac{M_{1}\left(m_{3}-m_{2}\right)}{v^{2}} .
$$

We first note that an even stronger bound applies to $\left|\epsilon_{N_{2}}\right|$ :

$$
\left|\epsilon_{N_{2}}\right| \leq \frac{4}{3} \frac{M_{1}}{M_{2}}\left[\ln \left(\frac{M_{2}}{M_{1}}\right)-1\right] \epsilon^{\mathrm{DI}} .
$$

Second, we note that $m_{3}-m_{2}$ is fixed to either $m_{a}-m_{s}$ $(\mathrm{NH})$ or $m_{s}^{2} /\left(2 m_{a}\right)(\mathrm{IH})$ [15], and so we can be more specific in Eq. (26):

$$
\epsilon^{\mathrm{DI}}= \begin{cases}M_{1} /\left(2.5 \times 10^{16} \mathrm{GeV}\right) & \mathrm{NH} \\ M_{1} /\left(10^{18} \mathrm{GeV}\right) & \mathrm{IH}\end{cases}
$$

In addition, since for $\mathrm{NH}(\mathrm{IH}) \tilde{m}_{1} \geq m_{s}\left(m_{a}\right)$, we have $\eta_{11} \leq 0.04(0.006)$. The upper bounds on $\epsilon_{N_{1}}$ and on $\eta_{11}$ give lower bounds on $M_{1}$,

$$
M_{1} \gtrsim \begin{cases}3.6 \times 10^{10} \mathrm{GeV} & \mathrm{NH} \\ 1.3 \times 10^{13} \mathrm{GeV} & \mathrm{IH}\end{cases}
$$

Alternatively, one can write upper bounds on $\tilde{m}_{1}$ :

$$
\tilde{m}_{1} \lesssim \begin{cases}m_{s} \times\left[M_{1} /\left(3.6 \times 10^{10} \mathrm{GeV}\right)\right]^{0.86} & \mathrm{NH} \\ m_{a} \times\left[M_{1} /\left(1.3 \times 10^{13} \mathrm{GeV}\right)\right]^{0.86} & \mathrm{IH}\end{cases}
$$

The $3+3$ framework. Within the $3+3$ framework, we can distinguish three different types of light neutrino spectra, Normal hierarchy $(\mathrm{NH})$, inverted hierarchy (IH), and quasi degeneracy (QD):

$$
\begin{aligned}
& m_{1} \ll m_{s}, \quad m_{2} \approx m_{s}, \quad m_{3} \approx m_{a}(\mathrm{NH}) ; \\
& m_{1} \ll m_{a}, \quad m_{2,3} \approx m_{a}, \quad m_{3}-m_{2}=\frac{m_{s}^{2}}{2 m_{a}}(\mathrm{IH}) ; \\
& m_{i} \approx \bar{m} \gg m_{a}, \quad m_{3}-m_{2}=\frac{m_{a}^{2}}{2 \bar{m}}, \quad m_{2}-m_{1}=\frac{m_{s}^{2}}{2 \bar{m}} \quad(\mathrm{QD}) .
\end{aligned}
$$


Using Eq. (9), we obtain lower bounds on the washout parameters (the first relation was derived in Ref. [16]):

$$
\begin{aligned}
\tilde{m}_{\alpha} & \geq m_{1}, \\
\tilde{m}_{1}+\tilde{m}_{2}+\tilde{m}_{3} & \geq m_{1}+m_{2}+m_{3} .
\end{aligned}
$$

Evaluating Eqs. (13), we obtain

$$
\begin{gathered}
m_{1}^{2}+m_{2}^{2}+m_{3}^{2}=\tilde{m}_{1}^{2}+\tilde{m}_{2}^{2}+\tilde{m}_{3}^{2}+2 \mathcal{R} e\left(\tilde{m}_{12}^{2}+\tilde{m}_{23}^{2}+\tilde{m}_{13}^{2}\right) \\
m_{1}^{2} m_{2}^{2}+m_{1}^{2} m_{3}^{2}+m_{2}^{2} m_{3}^{2}=\left(\tilde{m}_{11} \tilde{m}_{22}-\left|\tilde{m}_{12}\right|^{2}\right)^{2} \\
+\left(\tilde{m}_{11} \tilde{m}_{33}-\left|\tilde{m}_{13}\right|^{2}\right)^{2}+\left(\tilde{m}_{22} \tilde{m}_{33}-\left|\tilde{m}_{23}\right|^{2}\right)^{2} \\
+2 \mathcal{R} e\left[\left(\tilde{m}_{11} \tilde{m}_{23}^{*}-\tilde{m}_{12} \tilde{m}_{31}\right)^{2}\right. \\
\left.+\left(\tilde{m}_{22} \tilde{m}_{31}^{*}-\tilde{m}_{23} \tilde{m}_{12}\right)^{2}+\left(\tilde{m}_{33} \tilde{m}_{12}^{*}-\tilde{m}_{31} \tilde{m}_{23}\right)^{2}\right] \\
m_{1} m_{2} m_{3}=\tilde{m}_{11} \tilde{m}_{22} \tilde{m}_{33}-\tilde{m}_{33}\left|\tilde{m}_{12}\right|^{2}-\tilde{m}_{22}\left|\tilde{m}_{13}\right|^{2} \\
\quad-\tilde{m}_{11}\left|\tilde{m}_{23}\right|^{2}+2 \mathcal{R} e\left(\tilde{m}_{12} \tilde{m}_{23} \tilde{m}_{31}\right) \\
\leq \tilde{m}_{1} \tilde{m}_{2} \tilde{m}_{3} .
\end{gathered}
$$

Using the fact that $\tilde{m}$ is hermitian and positive, and the general property that for any positive definite matrix $A$ one has $\operatorname{Tr}\left[\left(A A^{\dagger}\right)^{-1 / 2}\right] \geq \operatorname{Tr}\left[\left(A A^{*}\right)^{-1 / 2}\right][17]$, we obtain

$$
\begin{aligned}
\tilde{m}_{1} \tilde{m}_{2}+\tilde{m}_{2} \tilde{m}_{3} & +\tilde{m}_{3} \tilde{m}_{1}-\left(m_{1} m_{2}+m_{2} m_{3}+m_{3} m_{1}\right) \\
& \geq\left|\tilde{m}_{12}\right|^{2}+\left|\tilde{m}_{23}\right|^{2}+\left|\tilde{m}_{13}\right|^{2}
\end{aligned}
$$

Eqs. (33) and (36) can be combined to give

$$
\begin{aligned}
\tilde{m}_{1}^{2} & +\tilde{m}_{2}^{2}+\tilde{m}_{3}^{2}-2\left(\tilde{m}_{1} \tilde{m}_{2}+\tilde{m}_{1} \tilde{m}_{3}+\tilde{m}_{2} \tilde{m}_{3}\right) \\
& \leq m_{1}^{2}+m_{2}^{2}+m_{3}^{2}-2\left(m_{1} m_{2}+m_{1} m_{3}+m_{2} m_{3}\right) .
\end{aligned}
$$

We now use the above equations and inequalities to obtain lower bounds on the $\tilde{m}_{\alpha}$ 's. We denote by $\tilde{m}_{a}, \tilde{m}_{b}, \tilde{m}_{c}$ the smallest, intermediate and largest $\tilde{m}_{\alpha}$, respectively. The left hand side of the inequality (37) is minimized for $\tilde{m}_{b}-\tilde{m}_{a}=0$. It is maximized (for $\tilde{m}_{c} \geq 2\left(\tilde{m}_{b}+\tilde{m}_{a}\right)$ ) by maximal $\tilde{m}_{c}$ which, according to Eq. (32), is given by $\sum_{i} m_{i}-\left(\tilde{m}_{b}+\tilde{m}_{a}\right)$. We can then write an inequality that depends on the sum of the two smallest $\tilde{m}_{\alpha}$ :

$$
3\left(\tilde{m}_{a}+\tilde{m}_{b}\right)^{2}-4 \sum_{i} m_{i}\left(\tilde{m}_{a}+\tilde{m}_{b}\right)+4 \sum_{i<j} m_{i} m_{j} \leq 0,
$$

This inequality leads to an interesting lower bound,

$$
\tilde{m}_{a}+\tilde{m}_{b} \geq \frac{2}{3} \sum_{i} m_{i}-\frac{2}{3}\left(\sum_{i} m_{i}^{2}-\sum_{i<j} m_{i} m_{j}\right)^{1 / 2} .
$$

This bound has interesting implications for models of $N_{2}$ leptogenesis that are based on $\tilde{m}_{1}$ in the weak washout region [18, 19, 20], where it gives $\tilde{m}_{2} \gtrsim m_{s}$. (A qualitative statement in this regard was made in ref. [19].)

Eqs. (31), (32) and (39) lead to the following lower bounds:
- Normal hierarchy:

$\tilde{m}_{c} \geq \frac{m_{a}}{3}\left(1+\frac{m_{s}}{m_{a}}\right), \quad \tilde{m}_{b} \geq \frac{m_{s}}{2}\left(1-\frac{m_{s}}{4 m_{a}}\right), \quad \tilde{m}_{a} \geq m_{1}$.

- Inverted hierarchy:

$$
\tilde{m}_{c} \geq \frac{2 m_{a}}{3}, \quad \tilde{m}_{b} \geq \frac{m_{a}}{2}, \quad \tilde{m}_{a} \geq m_{1} .
$$

- Quasi degeneracy:

$$
\tilde{m}_{\alpha} \geq \bar{m}
$$

We conclude that, for hierarchical (quasi-degenerate) light neutrino masses, at least two (all three) of the $\tilde{m}_{\alpha}$ are in the strong washout region.

Conclusions. We investigated the relations between leptogenesis parameters and light neutrino masses. In particular, we derived exact relations between elements of the $\tilde{m}$ matrix [defined in Eq. (8)], relevant to leptogenesis, and the light neutrino masses. The diagonal elements, $\tilde{m}_{\alpha}$, determine the $\Delta L=1$ washout effects. As concerns the off-diagonal ones, $\mathcal{I} m\left(\tilde{m}_{\alpha \beta}\right)$ determine the size of the CP asymmetries, while $\left|\tilde{m}_{\alpha \beta}\right|$ is related to projections (in heavy flavor space) of the asymmetries generated by heavy singlet neutrinos due to interactions of lighter singlets.

The resulting equations lead to interesting exact relations, such as Eq. (23), between the washout parameters, $\mathrm{CP}$ asymmetries and neutrino masses. The various relations lead to simple inequalities between the washout parameters $\tilde{m}_{\alpha}$ and the light neutrino masses $m_{i}$, see Eqs. (17) - (21) for the $3+2$ framework and (31)-(39) for the $3+3$ framework.

For light neutrino masses with normal hierarchy, we find the following results:

- In the $3+2$ framework, both $N_{1}$ and $N_{2}$ interactions are in the strong washout region, with both $\tilde{m}_{\alpha} \geq$ $0.009 \mathrm{eV}$ and at least one $\geq 0.025 \mathrm{eV}$.

- In the $3+3$ framework, at least two $N_{\alpha}$ 's have interactions in the strong washout region, with $\tilde{m}_{\alpha} \geq 0.005 \mathrm{eV}$ and at least one $\geq 0.02 \mathrm{eV}$.

The lower bounds are stronger for inverted hierarchy, and even more so in the $3+3$ framework with quasi-degenerate light neutrinos.

Acknowledgments. We thank Micha Berkooz, Oded Kenneth, Enrico Nardi, Esteban Roulet, Adam Schwimmer and Alessandro Strumia for useful discussions. This project was supported by the Albert Einstein Minerva Center for Theoretical Physics. The work of Y.G. is supported in part by the Israel Science Foundation under Grant No. 378/05. The research of Y.N. is supported by grants from the Israel Science Foundation 
founded by the Israel Academy of Sciences and Humanities, the United States-Israel Binational Science Foundation (BSF), Jerusalem, Israel, the German-Israeli foundation for scientific research and development (GIF), and the Minerva Foundation.

* Electronic address: guy.engelhard@weizmann.ac.il

$\dagger$ Electronic address: yuvalg@physics.technion.ac.il

‡ Electronic address: yosef.nir@weizmann.ac.il

[1] M. Fukugita and T. Yanagida, Phys. Lett. B 174, 45 (1986).

[2] R. Barbieri, P. Creminelli, A. Strumia and N. Tetradis, Nucl. Phys. B 575, 61 (2000) arXiv:hep-ph/9911315.

[3] A. Strumia, arXiv:hep-ph/0608347

[4] G. Engelhard, Y. Grossman, E. Nardi and Y. Nir, arXiv:hep-ph/0612187.

[5] J. A. Casas and A. Ibarra, Nucl. Phys. B 618 (2001) 171 arXiv:hep-ph/0103065.

[6] T. Endoh, T. Morozumi and Z. h. Xiong, Prog. Theor. Phys. 111, 123 (2004) arXiv:hep-ph/0308276.

[7] E. Nardi, Y. Nir, E. Roulet and J. Racker, JHEP 0601, 164 (2006) arXiv:hep-ph/0601084.

[8] A. Abada, S. Davidson, F. X. Josse-Michaux, M. Losada and A. Riotto, JCAP 0604, 004 (2006)
arXiv:hep-ph/0601083.

[9] W. Buchmuller, P. Di Bari and M. Plumacher, Nucl. Phys. B 643, 367 (2002) arXiv:hep-ph/0205349.

[10] G. F. Giudice, A. Notari, M. Raidal, A. Riotto and A. Strumia, Nucl. Phys. B 685, 89 (2004) arXiv:hep-ph/0310123.

[11] A. Ibarra and G. G. Ross, Phys. Lett. B 591, 285 (2004) arXiv:hep-ph/0312138.

[12] L. Covi, E. Roulet and F. Vissani, Phys. Lett. B 384, 169 (1996) arXiv:hep-ph/9605319.

[13] S. Davidson and A. Ibarra, Phys. Lett. B 535, 25 (2002) arXiv:hep-ph/0202239.

[14] T. Hambye, Y. Lin, A. Notari, M. Papucci and A. Strumia, Nucl. Phys. B 695, 169 (2004) arXiv:hep-ph/0312203.

[15] P. H. Chankowski and K. Turzynski, Phys. Lett. B 570, 198 (2003) arXiv:hep-ph/0306059.

[16] M. Fujii, K. Hamaguchi and T. Yanagida, Phys. Rev. D 65, 115012 (2002) arXiv:hep-ph/0202210.

[17] O. Kenneth, private communication.

[18] O. Vives, Phys. Rev. D 73, $073006 \quad$ (2006) arXiv:hep-ph/0512160.

[19] P. Di Bari, Nucl. Phys. B 727, 318 (2005) arXiv:hep-ph/0502082.

[20] S. Blanchet and P. Di Bari, JCAP 0606, 023 (2006) arXiv:hep-ph/0603107. 